\title{
GESTÃO ESTRATÉGICA DE PESSOAS E COMPROMETIMENTO ORGANIZACIONAL EM ORGANIZAÇÕES HOSPITALARES
}

\author{
STRATEGIC MANAGEMENT OF PEOPLE AND \\ ORGANIZATIONAL COMMITMENT \\ IN HOSPITAL ORGANIZATIONS
}

\author{
Data de submissão: 28-05-2015 \\ Aceite: 10-03-2016 \\ Claudio Cesar Silva ${ }^{1}$ \\ Nildes Raimunda Pitombo Leite ${ }^{2}$ \\ Leonel Cezar Rodrigues ${ }^{3}$
}

\section{RESUMO}

Esta pesquisa, de abordagem qualitativa, baseou-se em estudos de casos múltiplos em organizações hospitalares. Sua contribuição residiu na compreensão de como a gestão estratégica de pessoas pode favorecer o comprometimento organizacional, tendo como sujeitos os gestores de organizações hospitalares, e na ampliação das discussões sobre o conceito de comprometimento organizacional. A coleta de dados foi realizada por meio de entrevistas em profundidade, aplicação de survey de experiência e pesquisa documental. Para o tratamento dos dados e a análise dos resultados, foram utilizadas as estratégias de análise de conteúdo, análise reflexiva, análise documental e, em consequência, a triangulação de dados. Os resultados demonstraram que, quando as políticas e práticas da gestão estratégica de pessoas são integradas às estratégias organizacionais, há uma tendência da área de gestão de pessoas de atuar mais direcionada ao modelo de comprometimento. Além disso, os aspectos centrais dos conceitos e das percepções sobre comprometimento organizacional, elaborados pelos gestores, destacaram o engajamento, a dedicação e a identificação com a organização, que são características da dimensão afetiva de comprometimento, não sendo observadas referências às dimensões normativa e instrumental.

Palavras-chave: Comprometimento Organizacional. Gestão Estratégica de Pessoas. Organizações Hospitalares.

\footnotetext{
1 Possui graduação em Administração pela Universidade Federal de Mato Grosso do Sul, UFMS, mestrado em Agronegócios pela Universidade Federal de Mato Grosso do Sul, UFMS e doutorado em Administração pela Universidade Nove de Julho, UNINOVE. Atualmente é Chefe da Coordenadoria de Educação Aberta e a Distância da UFMS e Coordenador da Universidade Aberta do Brasil (UAB), na UFMS. Campo Grande. Mato Grosso do Sul. Brasil. E-mail: claudiorai@uol.com.br

2 Possui graduação em Administração de Empresas pela Escola de Administração de Empresas da Bahia, EAEBA, mestrado em Administração pela Universidade Federal da Bahia, UFBA e doutorado em Administração pela Universidade de São Paulo, USP. Osasco. São Paulo. Brasil. E-mail: nildespitombo53@gmail.com

3 Possui graduação em Química pela Fundação Universidade Regional de Blumenau, FURB, mestrado em Administração da Tecnologia pela Vanderbilt University, VANDERBILT, Estados Unidos e doutorado em Administração Universitária pela Vanderbilt University, Estados Unidos. Atualmente é professor titular e pesquisador do Programa de Doutorado em Administração (PPGA/UNINOVE), Coordenador do Programa de Doutorado Interinstitucional em Administração da Universidade Nove de Julho e Coordenador de Relações Internacionais do PPGA/ UNINOVE. São Paulo. São Paulo. Brasil. E-mail: leonel@uninove.br
} 


\begin{abstract}
This research is characterized as a qualitative approach based on multiple-case studies in hospital organizations. These findings enhance our understanding of how the strategic management of people can foster organizational commitment, using managers of hospital organizations as subjects, and expanding discussions about the concept of organizational commitment. Data were collected using in-depth interviews, qualitative survey questionnaires and documentary research. Data management and analysis were performed using analysis strategies, reflective analysis, document analysis and, consequently, triangulation of data. Overall, these results show that when the policies and practices of strategic management of people are integrated into organizational strategies, there is a tendency of people management act more focused on commitment model. Moreover, the central aspects of the concepts and perceptions about organizational commitment drawn up by managers highlight the commitment, dedication and identification with the organization as affective dimension characteristics of commitment found in the literature. However, no references were found to normative and instrumental dimensions.
\end{abstract}

Keywords: Strategic management of people. Organizational Commitment. Hospital Organizations.

\title{
1 INTRODUÇÃO
}

Esta pesquisa surgiu da relevância e do interesse que, nos últimos anos, a gestão estratégica de pessoas e o comprometimento organizacional estão alcançando nas organizações, tanto no âmbito acadêmico quanto profissional (BARRETO et al., 2011; BASTOS et al., 2014; CALDAS et al., 2003; DEMO, 2003; KLEIN; MOLLOY; BRINSFIELD, 2012; MASCARENHAS; BARBOSA, 2013). Desde o final da década de 1980, Guest (2011) afirmava que a gestão de pessoas do século XXI deveria ter suas políticas e práticas de gestão integradas aos planos estratégicos das organizações, a fim de obter resultados efetivos para os negócios. Em consonância com esse autor, Albuquerque (2002) também reforçou o objetivo próprio da gestão estratégica de pessoas, que é o processo de busca do melhor alinhamento possível entre o comportamento das pessoas e as estratégias e os fins da organização, por intermédio da construção de uma cultura do comprometimento organizacional que responsabilize as pessoas na organização pela implantação da estratégia e pelo desempenho profissional.

Nesse contexto, esta pesquisa teve o intuito de contribuir para o avanço da ciência em relação aos estudos referentes à gestão estratégica de pessoas e ao comprometimento organizacional, conforme apontam as agendas nacionais e internacionais de pesquisas na área, relacionando esses fenômenos ao propósito de compreender e descrever suas inter-relações, em uma perspectiva qualitativa. Nessa concepção, definiu-se como objetivo principal investigar de que forma a gestão estratégica de pessoas pode contribuir para o comprometimento organizacional em organizações hospitalares. Para o alcance desse objetivo, estabeleceram-se como objetivos específicos: analisar a congruência entre o planejamento estratégico institucional e o planejamento da gestão de pessoas; identificar as políticas e práticas de gestão estratégica de pessoas que podem contribuir para o comprometimento organizacional; conhecer os conceitos e as percepções sobre o comprometimento organizacional atribuídos pelos gestores; e relacionar os conceitos de comprometimento organizacional dos gestores com as definições apresentadas pela literatura.

O campo de estudos foi constituído de organizações hospitalares, devido à importância social que essas organizações possuem e à escassez de trabalhos científicos acerca dessa temática, tendo como sujeitos de pesquisa gestores da alta direção e gestores intermediários. Assim, a opção pelas organizações hospitalares como campo de investigação e coleta de dados ocorreu em razão de essas organizações constituírem as principais engrenagens do sistema de prestação de serviços de saúde no Brasil (MELENCHION, 2006; OLIVEIRA, 2013). 


\section{GESTÃO ESTRATÉGICA DE PESSOAS}

Na gestão de pessoas, o conceito de gestão estratégica possui características próprias, conforme apresentaram Albuquerque (2002), Anthony, Perrewé e Kacmar (1996) e Mascarenhas (2011): focalizar o longo prazo; considerar todas as pessoas da organização; estar integrada com a estratégia corporativa e com as demais estratégias funcionais; reconhecer os impactos do ambiente organizacional externo; e reconhecer o impacto da competição e da dinâmica no mercado de trabalho.

No estudo das abordagens da gestão estratégica de pessoas, Albuquerque (2002) apresentou duas perspectivas estratégicas básicas de gestão de pessoas, a estratégia de controle e a estratégia de comprometimento. Na primeira, as pessoas são vistas como recursos de produção que necessitam ser controlados. Na segunda, as pessoas são consideradas parceiras da organização, de modo que o comprometimento com a organização está diretamente relacionado à melhoria do desempenho. Para esse autor, trata-se de diferentes filosofias de gestão, sem, entretanto, deixar de apontar a existência de uma tendência de evolução visando à mudança do modelo de controle para o de comprometimento.

Lacombe e Chu (2008), ao estudar a contribuição da gestão de pessoas para os resultados organizacionais, relataram que existem três tipos de estudos para teorizar a abordagem estratégica de gestão de pessoas: a universalista, a contingencialista e a configuracional. Nesse mesmo sentido, Mascarenhas (2011) analisou duas linhas de pensamento: gestão estratégica de pessoas como alinhamento sistêmico e gestão estratégica de pessoas como vantagem competitiva. Na visão de gestão estratégica de pessoas como alinhamento sistêmico, que tem como ideia principal a adoção de estruturas e estratégias específicas de cada organização para possibilitar a adaptação mais adequada ao seu ambiente e garantir melhor desempenho, destacam-se três abordagens teóricas: contingencialista, universalista e cultural.

A abordagem contingencialista pressupõe que, quanto mais alinhadas estiveram as estratégias e as práticas, maiores serão as possibilidades de desempenho superior da organização, o que indica uma forte relação entre as variáveis práticas de gestão de pessoas e estratégias organizacionais. Seguindo esse conceito, o modelo de gestão de pessoas deve: a) interagir com outras dimensões da organização para enfrentar os desafios impostos pelo ambiente; $b$ ) contribuir para os processos pelos quais a missão e os objetivos organizacionais são definidos; e c) estabelecer a utilização dos recursos disponíveis para atingi-los (LEGGE, 2006; MASCARENHAS, 2011).

$\mathrm{Na}$ abordagem universalista, estão incluídos estudos que defendem um conjunto de melhores práticas de gestão estratégica de pessoas que serviriam para todos os tipos de organizações. Essas práticas estratégicas podem ser relacionadas ao desempenho das organizações. Para Martín-Alcázar, Romero-Fernandez e Sánchez-Gardey (2005), a perspectiva universalista é a abordagem mais simples para a análise das estratégias de gestão de pessoas. Segundo esses autores, os pesquisadores podem, portanto, identificar as melhores práticas de gestão de pessoas para melhorar o desempenho organizacional. Seus estudos evidenciaram ainda que, na abordagem universalista, em sua fase inicial, as melhores práticas de gestão de pessoas eram orientadas para reforçar as habilidades e competências das pessoas, tais como: remuneração, alguns métodos de recrutamento e seleção, desenvolvimento continuado e avaliação de desempenho.

A abordagem cultural centrou-se nos aspectos da cultura organizacional, motivo pelo qual foi denominada de abordagem cultural da gestão estratégica de pessoas. Nessa abordagem, utilizam-se conceitos integrativos de cultura, com significados culturais amplamente compartiIhados e consensuais entre os membros do grupo, o que permite tratar a cultura como uma variável objetiva e manipulá-la em função de melhores desempenhos organizacionais (MASCARENHAS, 2011; STONE; STONE-ROMERO; LUKASZEWSKI, 2007). 
Já na visão de gestão estratégica de pessoas como vantagem competitiva, também denominada de abordagem configuracional, de acordo com Lacombe e Chu (2008) e Martín-Alcázar, Romero-Fernandez e Sánchez-Gardey (2005), o papel da gestão de pessoas transcende as barreiras da simples implementação de estratégias e assume a responsabilidade compartilhada pela formulação das estratégias organizacionais, em um processo dinâmico de geração de configurações de recursos e competências únicas, que, por sua vez, precisam ser articuladas por configurações de estratégias, políticas e práticas de gestão de pessoas, particulares para cada organização (ALBUQUERQUE, 2002; MASCARENHAS, 2011). Nessa abordagem, o sistema de gestão estratégica de pessoas é definido como um conjunto multidimensional de elementos que podem ser combinados de maneiras diferentes para obter um infinito número de configurações possíveis, conforme asseveraram Martín-Alcázar, Romero-Fernandez e Sánchez-Gardey (2005).

$\mathrm{Na}$ gestão estratégica de pessoas, as políticas e práticas podem ser mutuamente reforçadas para auxiliar no alcance dos objetivos organizacionais, fornecendo instrumentos para comunicar a visão estratégica e os objetivos da organização de forma que sejam mais facilmente compreendidos pelas pessoas (DEMO et al., 2011; OZOLINA-OZOLA, 2014). Em consonância com esses autores, Horta, Demo e Roure (2012) afirmaram que, mediante essas políticas de gestão de pessoas, as organizações procuram estabelecer comunicação com seus empregados, objetivando influenciar o seu comportamento e envolvimento no ambiente organizacional.

Com o avanço dos estudos acerca da gestão de pessoas, algumas pesquisas evidenciaram relações positivas entre políticas e práticas de gestão de pessoas com variáveis como comprometimento, desempenho, produtividade e qualidade (AYDOGDU; ASIKGIL, 2011; DEMO; MARTINS; ROURE, 2013; DEMO; ROZZETT, 2012; FRIMPONG, 2014; GUEST, 2011; KATOU, 2012; OZOLINA-OZOLA, 2014; VELD; PAAUWE; BOSELIE, 2010). Verificou-se, também, em estudos realizados por Bal, Kooij e De Jong (2013) e Boselie, Dietz e Boon (2005), que políticas e práticas de gestão de pessoas afetam favoravelmente o desempenho das organizações quando essas políticas e práticas são desenvolvidas de maneira articulada com as estratégias da organização. Para Bal, Kooij e De Jong (2013), que realizaram um estudo multinível junto a funcionários de organizações da área de saúde, as políticas e práticas de gestão de pessoas, no que tange aos aspectos das abordagens universalista e contingencialista, relacionam-se por meio de múltiplas vias com os resultados organizacionais e o comprometimento dos funcionários.

Para a consecução dos objetivos específicos desta pesquisa, optou-se por delimitar as políticas e práticas de gestão estratégica de pessoas voltadas para a promoção da valorização, do bem-estar e da realização profissional e pessoal, que, de acordo com Demo e Rozzett (2012), são políticas e práticas de: recrutamento e seleção; envolvimento; treinamento, desenvolvimento e educação; condições do trabalho; avaliação de desempenho e competências; e recompensas.

\section{COMPROMETIMENTO ORGANIZACIONAL}

Nas pesquisas sobre o construto comprometimento como vínculo de naturezas afetiva, instrumental e normativa, esse construto é estudado em função dos focos ou alvos que o trabaIhador tem, tais como: a organização, o grupo, o sindicato, os valores, a profissão e o trabalho (BASTOS et al., 2013; MEDEIROS; ENDERS, 2002). Vale ressaltar, ainda, que, de acordo com Menezes e Bastos (2011), dentro de cada foco (alvo) existem abordagens diferenciadas quanto às bases (natureza). São duas as principais abordagens sobre comprometimento organizacional: a atitudinal e a comportamental. A primeira focaliza o processo pelo qual as pessoas pensam a relação, as crenças, os afetos e as intenções com sua organização, desenvolvendo-se lentamente 
ao longo do tempo. A segunda considera que as pessoas se comprometem com ações, sendo as atitudes geradas pelos comportamentos (MENEZES; BASTOS, 2011).

Embora existam várias vertentes que abordem o construto comprometimento organizacional, elas compartilham a premissa de que o vínculo existe e de que significa o desejo de permanecer na organização, de exercer suas atividades e de acreditar e aceitar os objetivos e valores organizacionais (BANDEIRA; MARQUES; VEIGA, 2000; MEDEIROS; ENDERS, 2002). A linha de pesquisa que considera a natureza do vínculo como afetivo-atitudinal prevalece entre os pesquisadores, sendo essa abordagem aprimorada com os trabalhos de Mowday, Porter e Steers (1982). Para esses autores, o enfoque afetivo caracteriza-se como: uma forte crença e aceitação dos objetivos e valores da organização; uma tendência a manifestar um esforço considerável em favor da organização; e a manifestação de um forte desejo de permanecer como membro da organização. A partir dessa definição, verifica-se que sujeitos com elevado grau de comprometimento demonstram uma internalização dos valores e objetivos da organização e, por consequência, tendem a exercer um esforço considerável em favor dela.

O enfoque instrumental constitui o segundo referencial teórico adotado nas pesquisas sobre o tema, cujas denominações são: calculativo, continuação e side-bets (BASTOS, 1994). Essa corrente se originou nos trabalhos de Becker (1960), que a traduziu como função da percepção do trabalhador quanto às trocas estabelecidas com a organização, de modo que o sujeito permanece na organização devido aos custos e benefícios associados à sua saída. O comprometimento instrumental assinala o reconhecimento da pessoa no que se refere aos custos (financeiros, psicológicos e físicos) e às raras oportunidades de encontrar outro emprego caso decida sair da organização. Nesse enfoque do comprometimento, o funcionário permanece devido às recompensas e aos custos relacionados à sua saída da organização. Em outras palavras, refere-se aos custos que representaria deixar a organização e ao investimento feito pelo sujeito em relação, por exemplo, ao tempo há que trabalha na organização ou ao status da instituição (MEDEIROS; ENDERS, 2002).

O enfoque normativo, por sua vez, constitui a totalidade das pressões normativas internalizadas para agir em direção a um caminho que permita alcançar os objetivos e interesses organizacionais. Essa visão do comprometimento, segundo Wiener (1982), teria o foco centrado nos controles de normas e regulamentos por parte das organizações, ou seja, na intenção de exercer controle sobre as ações das pessoas por meio dos valores e objetivos organizacionais. Tais pressões provêm, certamente, da imposição da ação do empregado e do seu comportamento na organização, o que significa que obedece à cultura da organização. $O$ enfoque normativo procura trabalhar, assim, 0 plano organizacional por meio da análise da cultura e do lado individual, averiguando os processos motivacionais e partindo do pressuposto de que o comportamento do sujeito é conduzido de acordo com o conjunto de normas que ele assume internamente (GONZÁLEZ; GUILLÉN, 2008).

Nesse tipo de comprometimento, desenvolve-se um forte sentimento de permanecer na instituição, como efeito de experimentar uma sensação de dívida, pelo fato de a organização ter dado uma oportunidade ou recompensa que foi valorizada pelo empregado. Então, o comprometimento normativo, como citado por Meyer e Allen (1991) e Meyer et al. (2012), é emocional porque o sujeito sente a obrigação de não deixar a organização, por estar em dívida com ela. A base do vínculo desse comprometimento se encontra, portanto, na introjeção de valores e padrões culturais da organização no sujeito (LEITE; ALBUQUERQUE, 2011; MOWDAY; PORTER; STEERS, 1982).

O surgimento dos modelos multidimensionais coloca em destaque que, apesar da importância do vínculo emocional do empregado com a organização, existem outros fatores que intervêm no desenvolvimento do comprometimento organizacional e que contribuem para que o laço entre sujeito e organização seja mais complexo. Diante disso, alguns autores utilizam expressões distintas 
para denominar esses fatores. Para Mowday, Porter e Steers (1982), tais fatores constituem tipologias do comprometimento; Becker (1960), entretanto, registra-as como bases do comprometimento. Contudo, para Meyer e Allen (1991) e Meyer et al. (2012), são componentes do comprometimento.

Todavia, o modelo de maior aceitação pelos pesquisadores foi o de Meyer e Allen (1991), que conceitua comprometimento em três componentes: afetivo, instrumental e normativo. O modelo de Meyer e Allen (1991) estabeleceu que o comprometimento de um empregado com a organização pode refletir vários graus dos três componentes. Bandeira, Marques e Veiga (2000) consideraram o modelo tridimensional mais coerente com os ambientes interno e externo apresentados no cenário globalizado, por estar em consonância com a complexidade do ser humano e as contínuas mudanças e transformações socioeconômicas.

Entretanto, a multidimensionalidade do construto tem sido alvo de críticas (KLEIN; MOLLOY; COOPER, 2009; MAIA; BASTOS, 2011; MOSCON, 2009; RODRIGUES; BASTOS, 2010; ROWE; BASTOS; PINHO, 2011; SOLINGER; OLFFEN; ROE, 2008). De acordo com Moscon (2009), o modelo multidimensional de Meyer e Allen (1991) é cercado de controvérsias e reúne dimensões do comprometimento que podem ser consideradas quase que antagônicas entre si. Nesse ponto, é importante destacar que a definição do conceito de comprometimento organizacional também tem sido alvo de grandes controvérsias (SOLINGER; OLFFEN; ROE, 2008), variando entre a concepção do vínculo passivo, que explica a permanência do sujeito, alheia à sua vontade, e a concepção do vínculo ativo, que se traduz em empenho extra, identificação, lealdade, trocas, compartilhamento de metas e motivação (RODRIGUES; BASTOS, 2010).

Em estudos recentes, Rodrigues e Bastos (2010) apresentaram um conjunto de argumentos em defesa da reestruturação e da maior delimitação do conceito de comprometimento com a retirada do componente de continuação ou instrumental. Segundo esses autores, a partir do final do século XX, com novas relações de trabalho estabelecidas, enxugamento das organizações e difusão do conceito de competências, a busca de permanência do empregado cede diante da necessidade de melhores desempenhos e resultados em favor da organização. Nesse caso, apresentaram uma perspectiva de ajuste do modelo tridimensional de Meyer e Allen (1997), destacando a dimensão de continuação como distinta do que se entende, atualmente, por comprometimento organizacional. Os autores propuseram uma revisão da multidimensionalidade do conceito e a retirada da base instrumental. Corroboraram essa linha de pensamento, os estudos de Maia e Bastos (2011) e Nascimento, Emmendoerfer e Gava (2014).

\section{PROCEDIMENTOS METODOLÓGICOS}

Nesta pesquisa, optou-se pela abordagem qualitativa, tendo em vista as considerações de pesquisadores como Bastos et al. (2014), Medeiros et al. (2005) e Ventura e Leite (2014), que constataram, por meio de levantamento bibliográfico em periódicos científicos nacionais e internacionais, um reduzido número de estudos com abordagem qualitativa sobre os construtos gestão estratégica de pessoas e comprometimento organizacional. De acordo com Medeiros et al. (2005), as pesquisas que exploram esses construtos seguem a linha escalar ou são estudos de casos descritivos, realizados por meio de abordagem quantitativa com análises estatísticas.

Nesse contexto, com base em Cooper e Schindler (2003), Martins e Theóphilo (2007) e Yin (2010), definiu-se como estratégia de pesquisa o estudo de casos múltiplos em duas organizações hospitalares, denominadas aqui de Hospital Caso A (HCA) e Hospital Caso B (HCB), como forma de garantir o anonimato solicitado pelos sujeitos da pesquisa. Antes, foi realizado um estudo de caso piloto que permitiu consolidar os instrumentos de coleta de dados. Os critérios específicos para seleção dos ca- 
sos estudados foram os seguintes: 1) hospitais privados ou filantrópicos, com ou sem fins lucrativos; 2) existência de planejamento estratégico institucional; e 3) estrutura organizacional de gestão de pessoas.

O HCA foi fundado em 1963 e em 2012 obteve a certificação de Acreditação Hospitalar, que é um método de avaliação dos recursos institucionais que busca garantir a qualidade da assistência por meio de padrões previamente definidos. Sua estrutura organizacional apresenta seis cargos de gestão estratégica e 20 cargos de gestão intermediária. Já o HCB foi fundado em 1928 e atualmente é considerado o maior e mais equipado hospital de sua região. Sua estrutura organizacional apresenta quatro cargos de gestão estratégica e 32 cargos de gestão intermediária.

Nesta pesquisa, foram utilizadas combinações de estratégias de coletas. Para tanto, selecionaram-se as estratégias mais recomendadas e adequadas aos propósitos desta pesquisa, de acordo com Cooper e Schindler (2003) e Yin (2010), tais como a) entrevistas em profundidade com a equipe de alta direção; b) aplicação de survey de experiência com os gestores intermediários; e c) pesquisa documental.

A principal estratégia para coleta de dados consistiu em entrevistas em profundidade, do tipo semiestruturadas, conduzidas com uso de roteiro, mas com a liberdade de acréscimo de novas questões pertinentes por parte dos pesquisadores, e aplicadas aos sujeitos da equipe de alta direção, sendo quatro diretores do HCA e seis diretores do HCB.

Optou-se por uma segunda fonte de evidências para abarcar os gestores dos demais níveis hierárquicos das organizações, a estratégia de coleta survey de experiência. A estratégia de coleta de dados denominada de survey de experiência encontra respaldo teórico em alguns autores como Cooper e Schindler (2003). Trata-se de um roteiro de questões semiestruturadas e abertas, aplicadas a um grupo específico de sujeitos, os quais, após as orientações feitas pelos pesquisadores, apresentam suas respostas e percepções, alinhadas às suas experiências. Nesta pesquisa, utilizouse essa estratégia de coleta em um grupo de cada caso estudado, e as respostas dos sujeitos foram apresentadas de forma individual. O questionário da survey de experiência foi aplicado na própria organização, durante reunião previamente agendada para esse fim, e contou com a participação de 12 sujeitos do HCA e 14 sujeitos do HCB, ocupantes de cargos de gestão intermediária.

A coleta de dados secundários consistiu em verificações de documentos fornecidos pelos hospitais e presentes em seus sítios. Entre os principais documentos coletados, encontram-se: estatuto, regimento e regulamento interno dos hospitais; organograma; materiais de comunicação social dos hospitais; planejamento estratégico; planejamento estratégico de recursos humanos; código de ética e de conduta dos hospitais; e documentos relativos às políticas e práticas de gestão de pessoas.

Tanto para a análise de dados das entrevistas quanto para a análise da survey de experiência e da pesquisa documental, foi utilizada a estratégia de análise de conteúdo com base em Bardin (2011) e Vergara (2006). O nível proposto de análise dos dados foi o organizacional. $\mathrm{Na}$ fase de pré-análise dos dados, foram feitas inicialmente leituras flutuantes de cada uma das transcrições das entrevistas, com o intuito de explorar o material e operacionalizar e sistematizar as ideias iniciais. Em seguida, procederam-se aos recortes dos fragmentos essenciais para posterior agrupamento na grade de categorias e subcategorias elencadas. A pré-análise das respostas obtidas com a aplicação da survey de experiência também passou pelo mesmo processo descrito para as entrevistas. As respostas foram transcritas para uma única planilha, contendo um número de ordem. Em seguida, efetuou-se a leitura flutuante de todo o material. Entretanto, a pré-análise dos dados levantados por meio da pesquisa documental, foi realizada somente após a conclusão de todo o tratamento dos dados das entrevistas em profundidade e da survey de experiência, tendo em vista que essa fonte de evidência era secundária e tinha como objetivo complementar informações em relação aos dados levantados e aos objetivos desta pesquisa. 
Tomando-se por base Vergara (2006), no primeiro momento do tratamento dos dados desta pesquisa, foi definida a grade de análise fechada para estabelecimento das categorias de análise, elegendo-se, a partir de então, as categorias norteadoras: congruência entre o planejamento institucional e o planejamento da gestão de pessoas, percepções sobre a gestão estratégica de pessoas e percepções sobre o comprometimento organizacional. À medida que essas categorias eram pertinentes aos objetivos e problemas da pesquisa, e mostravam-se alinhadas à fundamentação teórica, buscou-se identificar no material selecionado em cada fonte de evidência os elementos a serem integrados nessas categorias. No segundo momento de análise, optou-se pela apresentação de subcategorias das categorias norteadoras, consideradas como oriundas de grade aberta, como forma de retirar das entrevistas mais elementos para reflexão. Segundo Vergara (2006), essas novas subcategorias fazem parte da grade aberta. Ao estabelecer o conjunto final de categorias e considerar o possível rearranjo, formou-se então o que essa autora considera de grade mista de categorias.

Na sequência, procedeu-se à análise de conteúdo. A operacionalização da análise de conteúdo foi realizada manualmente pelos pesquisadores. O mesmo tratamento dado às entrevistas em profundidade e à survey de experiência foi efetuado com os dados coletados por meio da pesquisa de documentos dos hospitais.

Para operacionalizar e sistematizar a análise dos dados coletados por meio da pesquisa de documentos dos hospitais, elaborou-se um formulário intitulado Controle de Documentos Pesquisados. O objetivo desse formulário foi catalogar os documentos analisados de forma unitária e registrar os dados básicos do documento e os conteúdos pertinentes às categorias e subcategorias elencadas, a fim de complementar e até mesmo comparar e verificar os dados levantados por meio das entrevistas em profundidade e das surveys de experiência.

Por meio da análise reflexiva, resgataram-se a questão e os objetivos da pesquisa e confrontaram-se os resultados obtidos com as teorias que fundamentaram a investigação. No processo reflexivo desta pesquisa, foram interpretados os dados empíricos coletados. Na sequência, foi realizada uma interpretação teórica da interpretação empírica realizada na primeira fase, confrontando-se as análises com a questão norteadora e os objetivos da pesquisa, com base em Alvesson e Sköldberg (2000).

Para operacionalização da estratégia de triangulação de dados desta pesquisa, com base em Denzin e Lincoln (2000), foram adotadas as seguintes ações: comparar os dados coletados por meio das fontes de entrevista em profundidade, survey de experiência e pesquisa documental; identificar as possíveis diferenças e semelhanças entre os achados dessas fontes de evidência; avaliar as limitações metodológicas que puderam causar as divergências; avaliar as contribuições das divergências; confrontar os resultados obtidos com a fundamentação teórica da pesquisa; e formular as conclusões.

\section{ANÁLISE DOS DADOS E DISCUSSÃO DOS RESULTADOS DA PESQUISA}

No que diz respeito à categoria "alinhamento da gestão de pessoas com as estratégias organizacionais", a partir da triangulação de dados das fontes de evidências, constata-se que parte dos gestores estratégicos e parte dos gestores intermediários percebem a área de gestão de pessoas como alinhada com as estratégias organizacionais e compreendem o planejamento estratégico institucional e o planejamento da gestão de pessoas como congruentes. Outra parte desses gestores, contudo, percebe a referida área como estando em processo de alinhamento, sendo necessários ajustes. Entretanto, a análise documental demonstra que, nos dois estudos de caso, os planejamentos estratégicos institucionais são congruentes com os planejamentos da gestão de pessoas e que a área está alinhada com as estratégias organizacionais. 
Nessa discussão dos resultados, relembra-se que, teoricamente, para Albuquerque (2002) e Guest (2011), a gestão estratégica de pessoas tem um objetivo próprio, que é o processo de busca do melhor alinhamento possível entre o comportamento das pessoas e as estratégias e os fins da organização, de modo que deveria ter suas políticas e práticas de gestão interligadas aos planos estratégicos das organizações. De acordo com essas considerações, verifica-se, por meio das percepções dos gestores das organizações hospitalares estudadas e da análise documental, que a gestão de pessoas, de ambos os estudos de caso, tem atuação no nível estratégico. Isso significa que essa gestão coordena, em parceria com as demais áreas, os processos de planejamento e estabelecimento de políticas e práticas de gestão de pessoas voltadas para o alcance da visão, da missão, dos valores, dos objetivos e dos planos estratégicos dessas organizações hospitalares.

Com referência às semelhanças na subcategoria de "clareza da visão, da missão, dos valores, dos objetivos e das metas", em ambos os estudos de caso, observa-se que existe clareza e aceitação em relação a essas definições. Tanto a clareza quanto a aceitação podem ser explicadas pela forma participativa com que os processos de planejamento foram desenvolvidos. No caso do HCA, houve preparação técnica e comportamental para a realização do planejamento estratégico com a participação de todas as áreas e, posteriormente à sua conclusão, houve um trabalho sistemático de divulgação e discussão de resultados, ajustes e melhorias, com o objetivo de fortalecer o comprometimento com as estratégias definidas. Também no caso do HCB o processo de planejamento estratégico foi desenvolvido com a efetiva participação de gestores e funcionários de todas as áreas, havendo, por meio de reuniões sistemáticas de discussões sobre o planejamento, denominadas de "rodas de conversas", estímulo à elaboração, compreensão e aceitação das definições estabelecidas.

Como salientado por Albuquerque (2002), Caldas et al. (2003), Demo e Rozzett (2012), Lacombe e Chu (2008) e Martín-Alcázar, Romero-Fernández e Sanchez-Gardey (2005), a existência de clareza e aceitação da visão, da missão, dos valores, dos objetivos e das metas pode indicar que a área de gestão de pessoas está atuando dentro do que esses autores chamaram de tendência de evolução da área, visando à mudança do modelo de controle para o de comprometimento. Conforme Albuquerque (2002), na perspectiva do modelo de controle, as pessoas são vistas como recursos de produção e precisam ser controladas. Já na perspectiva do modelo de comprometimento, as pessoas são consideradas parceiras da organização. Nesse caso, o comprometimento está relacionado com o alcance dos objetivos organizacionais, com vistas ao melhor desempenho. Não se observam diferenças de percepções significativas nessa subcategoria, o que pode indicar que a gestão de pessoas, de ambos os estudos de caso, por meio de suas políticas e práticas de envolvimento, está atingindo um de seus objetivos em termos de reconhecimento, relacionamento, participação e comunicação, conforme preconizado por Demo (2003), Demo et al. (2011) e Horta, Demo e Roure (2012).

No que se refere à categoria de "percepções sobre a gestão estratégica de pessoas", a partir da triangulação de dados das fontes de evidências, na subcategoria de "políticas e práticas de recrutamento e seleção", verifica-se que, no caso do HCA, apenas os gestores estratégicos percebem o recrutamento interno como uma forma de promoção e valorização dos funcionários. Na percepção desses gestores, as experiências com recrutamento externo, tanto de profissionais locais quanto de profissionais de outras regiões do Brasil, não foram bem-sucedidas devido a problemas de falta de qualificação desses profissionais e à dificuldade de adaptação à cultura local por parte dos profissionais de outras regiões. Por sua vez, os gestores intermediários não manifestaram percepções acerca dessas políticas e práticas, o que pode indicar que não sejam muito percebidas nesse grupo como facilitadoras do comprometimento. No caso do HCB, verifica-se que os gestores estratégicos e intermediários destacam positivamente o processo de 
recrutamento e seleção feito por meio do ingresso qualificado, que abrange tanto os candidatos ao recrutamento externo quanto os funcionários que almejam promoção ou mudança de função.

Confrontando-se a triangulação de dados dessa subcategoria com a fundamentação teórica desta pesquisa, é possível compreender que os dados empíricos são consistentes com as orientações propostas por Demo e Nunes (2012), quando afirmaram que as políticas e práticas de recrutamento e seleção tratam de uma proposta articulada para procurar funcionários e selecioná-los e buscam harmonizar valores, interesses, expectativas e competências da pessoa com as características e demandas do cargo e da organização. Nesse sentido, as políticas e práticas de recrutamento e seleção verificadas nos estudos de caso podem contribuir positivamente para o comprometimento organizacional, quando realizadas de forma a escolher os candidatos com maior potencial de identificação com os objetivos e valores das organizações.

Como afirmado por Bandeira, Marques e Veiga (2000), em suas conclusões acerca de um estudo quantitativo, não é possível apontar tendências de que as políticas e práticas de recrutamento e seleção contribuem para o comprometimento. Entretanto, tais autores entendem que essas políticas e práticas têm procurado o refinamento técnico de modo a atrair e selecionar os candidatos mais propensos à assiduidade, ao bom desempenho e à identificação com os valores e objetivos da organização, o que foi observado nos estudos de caso desta pesquisa.

Na subcategoria de "políticas e práticas de envolvimento", no que se refere às semeIhanças, verifica-se que a comunicação aberta e a prática do feedback são mais percebidas pelos gestores em ambos os estudos de caso. No estudo de caso do HCA, as semelhanças entre as fontes de evidência consistem em: harmonização dos interesses da organização e das pessoas, valorização das pessoas, exemplo dos líderes, integração e confiança. Por sua vez, no estudo de caso do HCB, as semelhanças verificadas são: reconhecimento profissional e estímulo à participação.

Como enfatizado por Legge (2006), a área de gestão de pessoas tem o papel de contribuir para a implantação da estratégia da organização, no que diz respeito às dimensões humanas e sociais, o que a torna intermediária entre a alta direção e os demais grupos organizacionais. Nesse sentido, essas semelhanças observadas retratam que, nos estudos de caso do HCA e do $\mathrm{HCB}$, a gestão de pessoas tem buscado o desenvolvimento de práticas que melhorem os canais de comunicação interna; fortaleçam o clima de compreensão, cooperação e confiança entre gestores e subordinados e entre colegas; e fomentem a participação de todos nos processos de trabalho. Do ponto de vista dos gestores, essas políticas e práticas são contributivas para o comprometimento nas organizações hospitalares estudadas.

No que diz respeito às diferenças apresentadas pelos gestores, destacam-se as percepções dos gestores estratégicos do HCA, que compreendem que todo gestor é um gestor de pessoas, e dos gestores intermediários do $\mathrm{HCB}$, que consideram o exemplo dos gestores como importante para o comprometimento de toda a equipe. Como discutido por Albuquerque (2002), quando a gestão estratégica de pessoas assume uma estratégia de comprometimento, apresenta como premissa a ideia de que todos os funcionários são parceiros nas atividades de gestão estratégica, de forma que o papel do gestor, em todas as áreas, torna-se ainda mais relevante por estar em interação diária com seus funcionários. Ainda segundo Legge (2006), na perspectiva da gestão estratégica de pessoas, a área de gestão de pessoas na prática se responsabilizaria pela estruturação de sistemas gerenciais que reforçassem novos comportamentos desejados, e os gestores seriam os inspiradores desses funcionários. Portanto, a importância de servirem de exemplo pode se dar em função da atuação desses gestores como cogestores da área de gestão de pessoas, conforme verificado nos dados empíricos. Essa afirmação encontra respaldo em autores como Albuquerque (2002), Legge (2006) e Mascarenhas (2011), quando afirmaram que, na 
gestão estratégica de pessoas orientada ao comprometimento, o papel dos gestores é um dos itens mais relevantes para a estratégia de comprometimento.

No que se refere à subcategoria das "políticas e práticas de treinamento, desenvolvimento e educação", no que concerne às semelhanças, verifica-se que todos os grupos de gestores percebem positivamente essas políticas e práticas como contributivas para o comprometimento, destacando-se a educação permanente, percebida como um instrumento de educação, valorização, crescimento, amadurecimento e desenvolvimento. Tal contribuição é ainda percebida como proporcionadora das competências necessárias aos funcionários para a realização dos objetivos organizacionais de forma comprometida. Conforme asseveraram Bandeira, Marques e Veiga (2000), Demo e Rozzett (2012) e Lacombe e Chu (2008), as políticas e práticas de treinamento, desenvolvimento e educação fazem parte da dinâmica das organizações e influenciam o vínculo do funcionário com a organização, influência essa que pode ser atribuída ao sentimento de competência pessoal que o funcionário apresenta ao desempenhar suas atividades.

Entre as diferenças observadas, merecem destaque as afirmações dos gestores estratégicos do HCA sobre a implantação de infraestrutura adequada ao funcionamento e suporte da educação permanente e as afirmações dos gestores intermediários acerca do fato de que a educação permanente poderia focar mais os aspectos relacionados ao comprometimento. Essas percepções reforçam a importância que essas políticas e práticas possuem como geradoras de comprometimento.

No caso do HCB, as diferenças observadas também reforçam a percepção positiva acerca da educação permanente. Para os gestores estratégicos, a educação permanente é facilitadora do alinhamento entre os objetivos organizacionais e as pessoas da organização, pois essa educação colabora com o processo de compreensão, reflexão e aceitação de visão, missão, valores e objetivos organizacionais, promovendo o desenvolvimento de competências que melhorem o desempenho. Para os gestores intermediários, a oferta de treinamentos e capacitações deve sempre ser crescente para fazer face às demandas e necessidades de mudanças da sociedade contemporânea.

Avançando-se na discussão sobre as "políticas e práticas de condições de trabalho", no que concerne às semelhanças entre os achados nas diferentes fontes de evidência, de ambos os estudos de caso, observa-se que as boas condições de espaço físico e infraestrutura são valorizadas para a percepção de um ambiente harmonioso e agradável de trabalho, atendendo à necessidade de conforto. $\mathrm{O}$ oferecimento de assistência à saúde para o funcionário e sua família também é destacado. No caso do HCB especificamente, houve a percepção dos dois grupos de gestores com relação ao sentimento de identificação com a organização, proporcionado pelo uso de novos uniformes para todas as categorias.

Aludindo-se às diferenças observadas, destaca-se a valorização dos funcionários em relação à flexibilidade de horários de trabalho como um dos facilitadores para a permanência do vínculo do funcionário com a organização por maior tempo.

Com referência às "políticas e práticas de avaliação de desempenho e competências", no que concerne às semelhanças, verifica-se que apenas no estudo de caso do HCA os gestores destacaram a questão da avaliação de desempenho como base para a progressão funcional. Quanto às diferenças, observa-se no caso do HCB que não foram registradas percepções sobre essa subcategoria por parte dos gestores estratégicos, enquanto que os gestores intermediários acreditam que a avaliação de desempenho contribui para o comprometimento, mas de certa forma sugerem mudanças no atual sistema, de forma a melhorar os instrumentos e a abrangência da avaliação.

Quanto à subcategoria de "políticas e práticas de recompensas", naquilo que é semeIhante entre os estudos de caso, observa-se que os gestores percebem essas políticas como contributivas para o comprometimento. Entretanto, ao afirmarem que as recompensas financeiras 
devem ser associadas ao reconhecimento pessoal, tais como elogios e valorização pessoal, compreende-se, a partir da percepção desses gestores, que as políticas e práticas de recompensas podem influenciar positivamente a dimensão instrumental do comprometimento, gerando o vínculo passivo, conforme enfatizaram Rodrigues e Bastos (2010). Entretanto, essas mesmas políticas e práticas só podem influenciar a dimensão afetiva do comprometimento, gerando o vínculo ativo, se estiverem acompanhadas de ações voltadas ao reconhecimento pessoal e profissional.

Quanto às "percepções sobre o comprometimento organizacional", a triangulação de dados empíricos desta pesquisa mostra que os conceitos de comprometimento organizacional atribuídos pelos gestores, de acordo com Mowday, Porter e Steers (1982), estão relacionados ao enfoque afetivo de comprometimento organizacional, sendo caracterizados por expressões ligadas à identificação e ao empenho superior em favor da organização. Nota-se, ainda, que, nesses conceitos apresentados pelos gestores, manifestam-se sentimentos e comportamentos relacionados ao vínculo ativo com a organização, que indicam engajamento e empenho extras em benefício da organização, bem como sentimentos de identificação e afeto com a organização (MOWDAY; PORTER; STEERS, 1982; RODRIGUES; BASTOS, 2010).

Ainda nessa subcategoria de "conceitos de comprometimento", observa-se que as diferenças conceituais apresentadas pelos gestores, tais como torcer pela organização, sentir carinho, respeito e sinergia, retribuir da melhor forma possível os benefícios recebidos, ter confiança mútua, garra e determinação, sentir prazer e paixão pelo que se faz e apresentar identificação com a organização, por exemplo, são conceitos que também se caracterizam como enfoque afetivo do comprometimento.

Destaca-se que os conceitos que se referem ao cumprimento de obrigações entre o indivíduo e a organização poderiam ser relacionados ao enfoque instrumental, conforme apresentado por Becker (1960). Entretanto, os gestores complementaram que o cumprimento das obrigações contratuais deve estar vinculado a um esforço superior em favor da organização, e não apenas ao cumprimento das obrigações contratadas. Nesse sentido, entende-se que os gestores não percebem como comprometimento organizacional o fato de o indivíduo permanecer na organização e cumprir seu papel contratado se não houver identificação com os valores e objetivos organizacionais e comportamento de engajamento.

Os resultados desta pesquisa, no que se refere à subcategoria de "conceitos de comprometimento", mostram que esse construto é mais percebido pelas suas características afetivas. Não foram observadas, nos conceitos elaborados pelos gestores participantes desta pesquisa, evidências de elementos característicos da dimensão instrumental, constante do modelo multidimensional proposto por Meyer e Allen (1991), que se caracteriza pela questão da permanência ou saída da organização em função de uma necessidade pessoal. Ressalta-se que os relatos dos gestores participantes desta pesquisa, na subcategoria "o que não é ser comprometido", indicam que os comportamentos característicos dessa dimensão instrumental do construto são associados de forma negativa ao vínculo do indivíduo com a organização, sendo apontados, portanto, como características de um indivíduo descomprometido. Esses resultados encontram respaldo nos estudos realizados por Bastos, Brandão e Pinho (1997), Moscon (2009), Rodrigues e Bastos (2010) e Rowe, Bastos e Pinho (2011), que defenderam a revisão do conceito multidimensional de comprometimento, devido à sobreposição das dimensões afetiva e normativa, e a retirada da dimensão instrumental.

No que tange às percepções dos gestores apresentadas na subcategoria "o que é ser comprometido com a organização", verificam-se como semelhanças as seguintes características do comprometimento de base afetiva: identificação, incluindo sentimentos de afeto pela organização, proatividade e busca de melhores resultados como comportamentos de dedicação em favor da organização. Entre as diferenças, observa-se que as percepções "do que é ser compro- 
metido com a organização" também reforçam que, para esses gestores, o comprometimento organizacional está relacionado à dimensão afetiva e apresenta um vínculo ativo do indivíduo com sua organização. As percepções desses gestores encontram correspondência nos trabalhos de pesquisadores como Bastos, Brandão e Pinho (1997), que apresentaram como aspectos centrais do conceito de comprometimento o engajamento, a dedicação e o zelo pela organização, entre outras características que englobam a percepção de vínculo ativo do comprometimento.

Na subcategoria "o que não é ser comprometido com a organização", verifica-se que as percepções dos gestores no que concerne às semelhanças observadas nos dois estudos de caso (tais como "apenas cumprir as obrigações", "ser indiferente" e "não ser proativo", comportamentos interpretados como pertencentes àquele que executa seu papel sem oferecer algo a mais, no sentido de melhorar processos e desempenhos) são representadas pelas características do vínculo passivo de comprometimento organizacional, o qual, de acordo com Mowday, Porter e Steers (1982), Rodrigues e Bastos (2010) e Solinger, Olffen e Roe (2008), caracteriza-se pelas ideias de permanência na organização, mas sem os laços de engajamento, identificação e afeto. Ainda na observação das semelhanças, nota-se que, para os gestores do HCA, aquele que não é comprometido com a organização apresenta os comportamentos de impontualidade e absenteísmo, caracterizados como negativos e/ou, como entendem Solinger, Olffen e Roe (2008), denominados de comportamentos destrutivo-passivos.

Ressalta-se ainda que, quanto às diferenças observadas, destacam-se as percepções dos gestores estratégicos do HCA e do HCB, ao considerarem que aquele que não é comprometido com a organização apenas realiza o que se espera em troca da remuneração; não oferece algo a mais; cumpre um contrato em troca dos benefícios oferecidos; e pode não se identificar nem tão pouco oferecer empenho extra pela organização. Nesse caso, para esses gestores, o que a literatura aponta como comprometimento organizacional instrumental não é percebido como comprometimento organizacional (BASTOS, 1994; BASTOS; BRANDÃO; PINHO, 1997; BECKER, 1960).

No que se refere aos estudos de casos desta pesquisa, nota-se que os gestores, a partir de vivência, formação, desenvolvimento, interação profissional e observações, acabam por elaborar seus próprios conceitos e teorias acerca do comprometimento. Nesta pesquisa, no que concerne aos aspectos centrais dos conceitos de comprometimento elaborados pelos gestores e das caracterizações "do que é ser comprometido" e "do que não é ser comprometido" com a organização, destacam-se o engajamento, a dedicação e a identificação com a organização, características típicas da dimensão afetiva e do vínculo ativo com a organização, enquanto que as demais dimensões, normativa e instrumental, não foram percebidas como dimensões de comprometimento organizacional, tal como a literatura apresenta. Essa conclusão encontra respaldo em alguns autores, como Rodrigues e Bastos (2010), que sugeriram maior discussão a respeito da retirada da dimensão instrumental do construto, uma vez que o simples fato de permanecer na organização não significa necessariamente se comprometer com ela.

Quanto à dimensionalidade desse construto, as evidências empíricas encontradas nesta pesquisa estão condizentes com os estudos que questionam a permanência da dimensão instrumental como integrante do núcleo central do conceito de comprometimento organizacional. Os participantes não fazem qualquer associação positiva entre o comprometimento e as questões pertinentes a essa dimensão instrumental, uma vez que os relatos registrados indicam claramente que tais peculiaridades são associadas ao que não é ser comprometido. Dessa forma, os resultados encontrados permitem apontar a necessidade de revisão da multidimensionalidade do construto e a possibilidade da retirada da dimensão instrumental, especialmente no que se refere ao modelo tridimensional proposto por Meyer e Allen (1991). Nesse sentido, outros pes- 
quisadores, como Bastos, Brandão e Pinho (1997), Klein, Molloy e Cooper (2009), Rodrigues e Bastos (2010) e Rowe e Bastos (2010), propuseram a revisão do modelo tridimensional de Meyer e Allen (1991), com a retirada da dimensão instrumental de comprometimento, e sugeriram a possibilidade de tratar o comprometimento como um construto unidimensional.

\section{CONCLUSÕES E RECOMENDAÇÕES}

Salienta-se que as considerações aqui apresentadas se referem exclusivamente aos estudos de caso analisados. Desse modo, esta pesquisa pôde ratificar que a gestão estratégica de pessoas se caracteriza, principalmente, pela busca do melhor alinhamento possível entre o comportamento das pessoas, as estratégias e os fins da organização, corroborando a máxima de que, quando as políticas e práticas da gestão estratégica de pessoas são integradas às estratégias organizacionais, verifica-se uma tendência na área de gestão de pessoas de atuar mais direcionada ao modelo de comprometimento. Os achados desta pesquisa também mostraram que é possível confirmar, entre as fundamentações teórica e empírica, que o construto comprometimento organizacional é composto tanto de elementos característicos da natureza atitudinal, que abrange as crenças, os afetos e as intenções, quanto de indicadores de intenções comportamentais, que focam as atitudes geradas pelo comprometimento.

As limitações deste estudo estão diretamente ligadas à metodologia qualitativa adotada. Para minimizá-las, foram tomados determinados cuidados, com base na fundamentação metodológica escolhida. Com referência ao problema da contaminação devido à interpretação dos pesquisadores, utilizou-se a perspectiva de triangulação de dados. Na fase de análise dos dados empíricos, foi submetido o rascunho das conclusões aos próprios gestores participantes, oriundos das organizações hospitalares, para que validassem os resultados. Quanto à questão do potencial de generalização, buscou-se realizar um estudo multicasos, de natureza exploratória, em que se procuram respostas, sem o objetivo de testar um quadro teórico. Com relação à confiabilidade, elaborou-se um protocolo de estudo de caso de forma a sistematizar todo o conjunto de dados coletados e analisados.

Por outro lado, esta pesquisa apresenta contribuições para a comunidade científica, as organizações hospitalares, os sujeitos participantes e a academia. Para a comunidade científica, destaca-se a compreensão de como as políticas e práticas de gestão estratégica de pessoas podem contribuir para o comprometimento organizacional, a partir de uma perspectiva qualitativa, em que se buscou a compreensão dos fenômenos com base nas percepções dos gestores que lideram o cotidiano das organizações hospitalares estudadas. Para essas organizações hospitalares, destaca-se a contribuição que o suporte teórico e a análise dos dados empíricos aqui apresentados pode oferecer, de modo a atender suas necessidades de implementar políticas e práticas de gestão de pessoas que possam ajudar no comprometimento organizacional. Essas organizações poderão desenvolver pesquisas internas que aprofundem e complementem os focos da gestão estratégica de pessoas que não foram contemplados nesta pesquisa. Para os gestores participantes desta pesquisa, a contribuição pode residir na oportunidade de reflexão acerca do papel fundamental que exercem junto às suas equipes, por meio de seus exemplos, suas vivências e suas experiências. No que diz respeito à academia, acredita-se que esta pesquisa contribui para os estudos sobre a gestão estratégica de pessoas e, também, para o refinamento do construto comprometimento organizacional.

Quanto à formulação de uma agenda para futuras pesquisas, sugere-se, no campo das organizações hospitalares, que sejam realizados estudos de caso comparativos entre as organizações hospitalares públicas e privadas, localizadas nas diversas regiões do Brasil e até mesmo estudos comparativos entre países, que possam ampliar as discussões considerando-se culturas 
diferentes. Já em relação aos aspectos metodológicos, sugere-se que outras abordagens e estratégias sejam utilizadas, contemplando a triangulação de dados, métodos e teorias, e que os sujeitos participantes e/ou amostra possam ser ampliados para outras categorias.

\section{REFERENCIAS}

ALBUQUERQUE, L. G. A gestão estratégica de pessoas. In: FLEURY, M. T. L. (Coord). As pessoas na organização. São Paulo: Gente, 2002. p. 35-50.

ALVESSON, M.; SKOLDBERG, K. Reflexive methodology: New vision for qualitative research. London: Sage Publications, 2000.

ANTHONY, W. P.; PERREWÉ, P. L.; KACMAR, K. M. Strategic resource management: $A$ Strategic Approach. [S.I.]: Harcourt Brace \& Co, 1996.

AYDOGDU, S.; ASIKGIL, B. An empirical study of the relationship among job satisfaction, organizational commitment and turnover intention. International Review of Management and Marketing, v. 1, n. 3, p. 4353, 2011.

BAL, P. M.; KOOIJ, D. T. A. M.; DE JONG, S. B. How do developmental and accommodative HRM enhance employee engagement and commitment? The role of psychological contract and SOC strategies. Journal of Management Studies, v. 50, n. 4, p. 545-572, 2013.

BANDEIRA, M. L.; MARQUES, A. L.; VEIGA, R. T. As dimensões múltiplas do comprometimento organizacional: um estudo na ECT/MG. Revista de Administração Contemporânea, v. 4, n. 2, p. 133-157, 2000.

BARDIN, L. Análise de conteúdo. São Paulo: Edições 70, 2011.

BARRETO, L. M. T. S. et al. Temas emergentes em gestão de pessoas: uma análise da produção acadêmica. Revista de Administração da UFSM, Santa Maria, v. 4, n. 2, p. 215-232, 2011.
BASTOS, A. V. B. Comprometimento no trabalho: a estrutura dos vínculos trabalhador com a organização, a carreira e o sindicato. 1994. 294 f. Tese (Doutorado em Psicologia) - Instituto de Psicologia, Universidade de Brasília, 1994.

BASTOS, A. V. B. et al. Comprometimento no trabalho: fundamentos para a gestão de pessoas. In: 0 trabalho e as organizações: atuações a partir da Psicologia. 1. ed. Porto Alegre: Artmed, 2013. p. 279-310.

BASTOS, A. V. B. et al. Vínculos dos indivíduos com a organização: análise da produção científica brasileira 2000-2010. Psicologia: Teoria e Pesquisa Revista, v. 30, n. 2, p. 153162, 2014.

BASTOS, A. V. B.; BRANDÃO, M. G. A.; PINHO, A. P. M. Comprometimento organizacional: uma análise do conceito expresso por servidores universitários no cotidiano de trabalho. Revista de Administração Contemporânea, v. 1, n. 2, p. 97-120, 1997.

BECKER, H. S. Notes on the concept of commitment. American journal of Sociology, v. 66, n. 1 , p. $32-40,1960$.

BOSELIE, P.; DIETZ, G.; BOON, C. Commonalities and contradictions in HRM and performance research. Human Resource Management Journal, v. 15, n. 3, p. 67-94, 2005.

CALDAS, M. et al. Produção acadêmica em recursos humanos no Brasil: 1991-2000. Revista de Administração de Empresas, v. 43, n. 1, p. 105-122, jan./fev./mar. 2003.

COOPER, D. R.; SCHINDLER, P. S. Métodos de pesquisa em administração, Porto Alegre: Bookman, 2003.

DEMO, G. Comprometimento no trabalho: uma síntese do estado da arte e uma revisão 
da produção nacional. Revista Psicologia, Organizações e Trabalho, Brasília, v. 3, n. 2, p. 185-213, jul./dez. 2003.

DEMO, G. et al. Políticas de gestão de pessoas no novo milênio: cenário dos estudos publicados nos periódicos da área de Administração entre 2000 e 2010. Revista de Administração Mackenzie, v. 12, n. 5, p. 15-42, 2011.

DEMO, G.; MARTINS, P. R.; ROURE, P. Políticas de gestão de pessoas, comprometimento e satisfação no trabalho na Livraria Cultura. Revista Alcance - Eletrônica, v. 20, n. 2, p. 237254, abr./jun. 2013.

DEMO, G.; NUNES, I. Políticas de gestão de pessoas: pressupostos, definições, resultados e produção internacional recente. In: DEMO, G. (Org.). Políticas de gestão de pessoas nas organizações: o estado da arte, produção nacional, agenda de pesquisa, medidas e estudos relacionais. São Paulo: Atlas, 2012. p. 33-48.

DEMO, G.; ROZZETT, K. Human resource management policies and practices (hrmpp): scale validation in the United States. International Journal of Strategic Management, v. 12, n. 3, 2012.

DENZIN, N. K.; LINCOLN, Y. S. Handbook of qualitative research. London: SAGE Publications, 2000.

FRIMPONG, D. O. Staff Satisfaction with Human Resource Management Practices at the University of Education, Winneba. European Journal of Business and Management, v. 6, $n$. 38, p. 87-95, 2014.

GONZÁLEZ, T. F.; GUILLÉN, M. Organizational commitment: A proposal for a wider ethical conceptualization of 'normative commitment'. Journal of Business Ethics, v. 78, n. 3, p. 401414, 2008.

GUEST, D. E. Human resource management and performance: still searching for some answers. HumanResource Management Journal, v. 21, n. 1, p. 3-13, 2011.

HORTA, P.; DEMO, G.; ROURE, P. Políticas de gestão de pessoas, confiança e bem-estar: estudo em uma multinacional. Revista de Administração Contemporânea, Rio de Janeiro, v. 16, n. 4, p. 566-585, jul./ago. 2012.

KATOU, A. A. Investigating reverse causality between human resource management policies and organizational performance in small firms. Management Research Review, v. 35, n. 2, p. 134-156, 2012.

KLEIN, H. J.; MOLLOY, J. C.; COOPER, J. T. Conceptual foundations: construct definitions and theoretical representations of workplace commitments. In: (Ed.). Commitment in organizations: Accumulated wisdom and new directions. 1. ed. New York: Taylor \& Francis, 2009. p. 3-36.

KLEIN, H. J.; MOLLOY, J. C.; BRINSFIELD, C. T. Reconceptualizing workplace commitment to redress a stretched construct: Revisiting assumptions and removing confounds. Academyof Management Review, v. 37, n. 1, p. 130-151, 2012.

LACOMBE, B. M. B.; CHU, R. A. Políticas e práticas de gestão de pessoas: as abordagens estratégica e institucional. Revista de Administração de Organizações, v. 48, n. 1, p. 25-35, 2008.

LEGGE, K. Humanresource management. In: ACKROYD, S.; BATT, R.; THOMPSON, P.; TOLBERT, P. S. (Ed.). The Oxford handbook of work and organization. New York: Oxford University Press, 2006.

LEITE, N. R. P.; ALBUQUERQUE, L. G. Gestão estratégica de pessoas, comprometimento e contrato psicológico: o caso Vale. Revista de Administração - RAUSP, São Paulo, v. 46, n. 1, p. 19-31, jan./mar. 2011.

MAIA, L. G.; BASTOS, A. V. B. Comprometimento calculativo e retaliação: visão integrada dos conceitos em uma organização pública. Revista 
de Administração da UFSM, Santa Maria, v. 4, n. 3, p. 390-405, set./dez. 2011.

MARTÍN-ALCÁZAR, F.; ROMERO-FERNÁNDEZ, P. M.; SÁNCHEZ-GARDEY, G. Strategic human resource management: integrating the universalistic, contingent, configurational and contextual perspectives. The International Journal of Human Resource Management, $v$. 16, n. 5, p. 633-659, 2005.

MARTINS, G. A.; THEÓPHILO, C. R. Metodologia da investigação científica para ciências sociais aplicadas. 1. ed. São Paulo: Atlas, 2007.

MASCARENHAS, A. O. Gestão Estratégica de Pessoas: evolução teoria e crítica. São Paulo: Cangage Learning, 2011.

MASCARENHAS, A. O.; BARBOSA, A. C. Q. B. Produção científica brasileira em gestão de pessoas no período 2000-2010. Revista de Administração de Organizações. São Paulo, v. 53, n. 1, p. 35-45, jan./fev. 2013.

MEDEIROS, C. A. F. et al. Um estudo exploratório dos múltiplos componentes do comprometimento organizacional. Revista eletrônica de administração, v. 11 , n. 1, p. 1-22, 2005.

MEDEIROS, C. A. F.; ENDERS, W. T. Comprometimento organizacional e características pessoais: como são os comprometidos e os descomprometidos com as organizações. Revista Eletrônica de Administração, v. 8, n. 5, 2002.

MELENCHION, J. R. Gestão de pessoas e gestão do conhecimento: conceituação e aplicabilidade nas organizações de saúde. $\mathbf{0}$ mundo da saúde, v. 30, n. 2, p. 215-227, abr./ jun. 2006.

MENEZES; I. G.; BASTOS, A. V. B. Comprometimento organizacional atitudinal: um estudo empírico sobre a dimensionalidade do construto. Estudos de Psicologia, Campinas, v. 28, n. 4, p. 463-474, out./dez. 2011.
MEYER, J. P. et al. Affective, normative, and continuance commitment levels across cultures: A meta-analysis. Journal of Vocational Behavior, v. 80, n. 2, p. 225-245, 2012.

MEYER, J. P.; ALLEN, N. J. A three-component conceptualization of organizational commitment. Human resource management review, v. 1, n. 1, p. 61-89, 1991.

MEYER, J. P.; ALLEN, N. J. Commitment in the workplace: theory, research, and application. London: Sage Publications, 1997.

MOSCON, D. C. B. Teorias implícitas de trabalhador comprometido e estratégias cotidianas de gestão: uma análise qualitativa. 2009. 136 f. Dissertação (Mestrado em Administração) - Universidade Federal da Bahia, Salvador, 2009.

MOWDAY, R. T.; PORTER, L. W.; STEERS, R. $M$. Employed organization linkages: the psychology of commitment, absenteism and turnover. New York: Academic Prest, 1982.

NASCIMENTO, A. L. D.; EMMENDOERFER, M.; GAVA, R. Comprometimento no trabalho de gestores públicos em uma autarquia estadual no Brasil. Revista do Serviço Público Brasília, v. 65, n. 2, p. 199-226, abr./jun. 2014.

OLIVEIRA, L. F. M. N. Gestão de pessoas em hospitais universitários: situação atual e tendências. 2013. 177 f. Tese (Doutorado em Gerenciamento em Enfermagem) Universidade de São Paulo, São Paulo, 2013.

OZOLINA-OZOLA, I. The Impact of Human Resource Management Practices on Employee Turnover. Procedia-Social and Behavioral Sciences, v. 156, p. 223-226, 2014.

RODRIGUES, A. C. A.; BASTOS, A. V. Problemas conceituais e empíricos na pesquisa sobre comprometimento organizacional: uma análise crítica do modelo tridimensional de J. Meyer e N. Allen. Revista Psicologia: Organizações e Trabalho, v. 10, n. 2, p. 129-144, jul./dez. 2010. 
ROWE, D. E. O.; BASTOS, A. V. B. Vínculos com a carreira e produção acadêmica: comparando docentes de IES públicas e privadas. Revista de Administração Contemporânea, Curitiba, v. 14, n. 6, p. 1011-1030, 2010.

ROWE, D. E. O.; BASTOS, A. V. B.; PINHO, A. P. $M$. Comprometimento e entrincheiramento na carreira: um estudo de suas influências no esforço instrucional do docente do ensino superior. Revista de Administração Contemporânea, v. 15, n. 6, p. 973-992, 2011.

SOLINGER, O. N.; OLFFEN, W.; ROE, R. A. Beyond the three-component model of organizational commitment. Journal of applied Psychology, v. 93, n. 1, p. 70-83, 2008.

STONE, D. L.; STONE-ROMERO, E. F.; LUKASZEWSKI, K. M. The impact of cultural values on the acceptance and effectiveness of human resource management policies and practices. Human resource management review, v. 17, n. 2, p. 152-165, 2007.

VELD, M.; PAAUWE, J.; BOSELIE, P. HRM and strategic climates in hospitals: does the message come across at the ward level? Human Resource Management Journal, v. 20, n. 4, p. 339-356, 2010.

VENTURA, V. L. S.; LEITE, N. P. Percepção da influência da gestão estratégica de pessoas no comprometimento organizacional. Revista Pretexto, v. 15, n. 3, p. 11-28, 2014.

VergarA, S. C. Métodos de Pesquisa em Administração. São Paulo: Atlas, 2006.

WIENER, Y. Commitment in organizations: a normative view. Academy of Management Review, v. 3, n. 7, p. 418-428, 1982.

YIN, R. K. Estudo de Caso: Planejamento e Métodos. 4. ed. Porto Alegre: Bookman, 2010. 